COMMUNICATIONS IN

ANALYSIS AND GEOMETRY

Volume 14, Number 3, 603-612, 2006

\title{
Ricci Curvature Rigidity for Weakly Asymptotically Hyperbolic Manifolds
}

\author{
Vincent Bonini $^{1}$, Pengzi MiaO ${ }^{2}$ And Jie Qing ${ }^{3}$
}

A rigidity result for weakly asymptotically hyperbolic manifolds with lower bounds on Ricci curvature is proved without assuming that the manifolds are spin. The argument makes use of a quasilocal mass characterization of Euclidean balls from [9] [14] and eigenfunction compactification ideas from [12].

\section{Introduction.}

Rigidity questions for asymptotically hyperbolic manifolds have been studied by many authors under various assumptions. In [10], Min-Oo proved a scalar curvature rigidity theorem for manifolds which are spin and are asymptotic to the hyperbolic space in a strong sense. In [1], Andersson and Dahl improved the scalar curvature rigidity for asymptotically locally hyperbolic spin manifolds. They also established the rigidity for conformally compact Einstein manifolds with spin structure. More recent related works are in [4], [15] and [16]. It is interesting to ask whether the spin structure is necessary to assure the rigidity. In [7], Listing was able to obtain a nonspin rigidity at the expense of replacing scalar curvature bound by sectional curvature bound. Very recently, in [12], Qing established the rigidity for conformally compact Einstein manifolds of dimension less than 7 without assuming spin structure. The proof in [12] uses conformal compactifications by positive eigenfunctions and the classic positive mass theorem proved by Schoen and Yau [13] for asymptotically flat manifolds. Based on ideas in [12] combined with a quasi-local mass characterization of Euclidean balls in [9], in this paper, we prove a Ricci curvature rigidity theorem for weakly asymptotically hyperbolic manifolds.

Theorem 1.1. Let $\left(X^{n+1}, g\right)$ be a weakly asymptotically hyperbolic manifold of order $C^{3, \alpha}$. Assume that $(X, g)$ has the standard round sphere $\left(S^{n},\left[h_{0}\right]\right)$ as its conformal infinity and satisfies Ric $(g) \geq-n g$. Let $r$ be the special defining function such that 


$$
g=\frac{1}{\sinh ^{2}(r)}\left\{d r^{2}+g_{r}\right\}
$$

in a neighbourhood of $\partial X$ and $g_{0}=h_{0}$. Then, if $2 \leq n \leq 6$ and

$$
\operatorname{Tr}_{g_{r}}\left(\frac{d}{d r} g_{r}\right) \in \Lambda_{0, \beta}^{s}(X)
$$

for some $s>1,(X, g)$ is isometric to the hyperbolic space $\mathbb{H}^{n+1}$.

We remark that Theorem 1.1 may be compared with the corresponding result in the asymptotically flat case established by Bartnik in [2]. The paper is organized as follows. In Section 2, we introduce notations and definitions. In Section 3, we recall some analytic and geometric preliminaries. In Section 4, we perform the conformal compactification and prove Theorem 1.1. We conclude the paper by comparing our result to scalar curvature rigidity for asymptotically hyperbolic manifolds in [1], [4], [10], [15] and [16].

\section{Weakly Asymptotically Hyperbolic Manifolds.}

In this section, we define our terms and introduce the function spaces that we will be working with. Throughout this paper, smooth will always mean $C^{\infty}$.

A smooth Riemannian metric $g$ in the interior $X^{n+1}$ of a smooth compact manifold $\bar{X}$ with boundary is said to be conformally compact of order $C^{m, \alpha}$ if $\bar{g}=\rho^{2} g$ extends as a $C^{m, \alpha}$ metric on $\bar{X}$, where $\rho$ is a smooth defining function for $M^{n}=\partial X$ in $\bar{X}$ in the sense that $\rho>0$ in $X$ and $\rho=0$, $d \rho \neq 0$ on $M$. The metric $\bar{g}$ restricted to $T M$ induces a metric $\hat{g}$ on $M$ which rescales upon change in defining function. Therefore, a conformally compact $\left(X^{n+1}, g\right)$ defines a conformal structure on $M$. We call $(M,[\hat{g}])$ the conformal infinity of $(X, g)$. When $m+\alpha \geq 2$, a straightforward computation as in [8] shows that the sectional curvatures of $g$ approaches $-|d \rho|_{\bar{g}}^{2}$ at $M$. Accordingly, we have the following definition for weakly asymptotically hyperbolic manifolds.

Definition 2.1. For a complete manifold $\left(X^{n+1}, g\right)$, we say the metric $g$ is weakly asymptotically hyperbolic of order $C^{m, \alpha}$ if $g$ is conformally compact of order $C^{m, \alpha}, m+\alpha \geq 2$ and $|d \rho|_{\bar{g}}^{2}=1$ along $M$.

To illustrate the difference between weakly asymptotically hyperbolic and asymptotically hyperbolic we recall, for instance, the following definition from $[15]$. 
Definition 2.2. A weakly asymptotically hyperbolic manifold $\left(X^{n+1}, g\right)$ is called asymptotically hyperbolic if it satisfies:

(1) The conformal infinity is the round sphere one $\left(S^{n},\left[h_{0}\right]\right)$.

(2) For a geodesic defining function $r$, we may write, in a collar neighbourhood of the infinity,

$$
g=\rho^{-2}\left(d r^{2}+g_{r}\right)
$$

where $\rho=\sinh r$,

$$
g_{r}=h_{0}+\frac{r^{n+1}}{n+1} h+O\left(x^{n+2}\right)
$$

and $h$ is a symmetric 2 -tensor on $S^{n}$.

A function $u$ which is $m$-times continuously differentiable on $X$ is said to be in the weighted Hölder space $\Lambda_{m, \alpha}^{s}(X)$ if $\|u\|_{m, \alpha}^{s}<\infty$ for $s \in R, m \geq 0$ and $\alpha \in(0,1)$, where the norm $\|u\|_{m, \alpha}^{s}$ is defined as follows. First, in the special case in which $X$ is a smoothly bounded open subset of $\mathbb{R}^{n+1}$, we define

$$
\|u\|_{m, 0}^{s}=\sum_{l=0}^{m} \sum_{|\gamma|=l}\left\|d^{-s+l} \partial^{\gamma} u\right\|_{L^{\infty}}
$$

and

$\|u\|_{m, \alpha}^{s}=\|u\|_{m, 0}^{s}+\sum_{|\gamma|=m} \sup _{x, y}\left[\min \left(d_{x}^{-s+m+\alpha}, d_{y}^{-s+m+\alpha}\right) \frac{\left|\partial^{\gamma} u(x)-\partial^{\gamma} u(y)\right|}{|x-y|^{\alpha}}\right]$,

where $d_{x}$ is the Euclidean distance from $x$ to $\partial X$. In the more general cases of a manifold with boundary, the same norms are defined using a covering by coordinate charts and a subordinate partition of unity in the usual way. We recommend [5] and [6] for succinct discussions of properties of the spaces $\Lambda_{m, \alpha}^{s}(X)$.

\section{Analytic and Geometric Preliminaries.}

We first, recall the following lemma from, for instance, [5] [6].

Lemma 3.1. Let $(X, g)$ be a weakly asymptotically hyperbolic manifold of order $C^{3, \alpha}$. Then, any representative $\hat{g}$ in the conformal infinity of $g$ determines a unique defining function $s \in C^{2, \alpha}(\bar{X})$ such that $\left.s^{2} g\right|_{T M}=\hat{g}, s^{2} g$ 
has a $C^{2, \alpha}$ extension to $\bar{X}$ and $|d s|_{s^{2} g}^{2} \equiv 1$ on a neighbourhood $U$ of $M$ in $\bar{X}$. Hence, $s$ determines an identification of $U$ with $M \times(0, \epsilon)$ such that

$$
g=\frac{1}{s^{2}}\left(d s^{2}+g_{s}\right)
$$

for a 1-parameter family $\left\{g_{s}\right\}$ of metrics on $M$ with $g_{0}=\hat{g}$.

By a change of variable

$$
s=\frac{\cosh (r)-1}{\sinh (r)}
$$

we may rewrite (3.1) as

$$
g=\rho^{-2}\left(d r^{2}+g_{r}\right)
$$

where $\rho=\sinh (r)$. One may compare (3.3) with the fact that

$$
g_{b}=\frac{1}{\sinh ^{2}(r)}\left\{d r^{2}+h_{0}\right\}
$$

gives the standard hyperbolic metric on $S^{n} \times \mathbb{R}^{+}$where $h_{0}$ is the standard metric on $S^{n}$. The fact that $s$ is $C^{2, \alpha}$ guarantees that the family of metrics $\left\{g_{r}\right\}$ is at least $C^{1}$ with respect to $r$. In the special case in which $\left(X^{n+1}, g\right)$ is Einstein and conformally compact of sufficiently high order, Andersson and Dahl [1] showed that the family of metrics $\left\{g_{r}\right\}$ in (3.3) have the properties

$$
g_{r}=h_{0}+\rho^{n} h, \operatorname{Tr}_{h_{0}} h=O\left(\rho^{n}\right), \rho=\sinh (r) .
$$

Thus, the decay assumption (1.2) is automatically satisfied by any conformally compact Einstein manifold with the round sphere as its conformal infinity. Next, we recall an analytic result of the operator $-\triangle_{g}+(n+1)$ between suitable weighted Hölder spaces (see Proposition 3.3 in [6]).

Lemma 3.2. Let $\left(X^{n+1}, g\right)$ be weakly asymptotically hyperbolic of order $C^{m, \alpha}$. Let $0<\beta<1$ and $k+1+\beta \leq m+\alpha$. Then,

$$
-\triangle+(n+1): \Lambda_{k+2, \beta}^{s} \rightarrow \Lambda_{k, \beta}^{s}
$$

is an isomorphism whenever $-1<s<n+1$.

In the final step of the proof in [12], the positive mass theorem is used on the doubling of a partially compactified manifold along its totally geodesic boundary. Here, we observe that it would be much simpler if we appeal to the following quasi-local mass type result proved in [9] [14](see also [11]). 
Proposition 3.3. Let $\bar{\Omega}^{n+1}$ be a smooth compact manifold with boundary $\partial \Omega$. Let $g$ be a metric on $\bar{\Omega}$ which is smooth in the interior $\Omega$ and $C^{2}$ up to $\partial \Omega$. If $g$ has non-negative scalar curvature in $\Omega,\left(\partial \Omega,\left.g\right|_{T \partial \Omega}\right)$ is isometric to $\left(S^{n}, h_{0}\right)$ and the mean curvature of $\partial \Omega$ with respect to the outward pointing unit normal identically equals the constant $n$, then $g$ has vanishing scalar curvature in $\Omega$ provided the dimension satisfies $2 \leq n \leq 6$.

Remark 3.4. It is desirable to further conclude that $g$ is actually flat on $\Omega$ which is indeed the case when $n=2[9]$. However, no proof in higher dimension is known so far except the case when $(\Omega, g)$ is assumed to be spin [14].

We conclude this section by recalling a nice functional characterization of the Hyperbolic space $\mathbb{H}^{n+1}$ proved in [11].

Lemma 3.5. Let $\left(X^{n+1}, g\right)$ be a complete Riemannian manifold. Assume that there exits a positive smooth function $u$ on $X$ such that

$$
H e s s_{g}(u)=u g .
$$

Then, $\left(X^{n+1}, g\right)$ is isometric to $\left(\mathbb{H}^{n+1}, g_{\mathbb{H}}\right)$.

\section{Proof of the Main Theorem.}

Let $\left(X^{n+1}, g\right)$ satisfy the assumptions in Theorem 1.1 and let $U$ be a neighbourhood of $M$ in $\bar{X}$ where (1.1) holds. We introduce a background hyperbolic metric

$$
g_{b}=\frac{1}{\rho^{2}}\left\{d r^{2}+h_{0}\right\}
$$

on $U$. Clearly, $\left(U, g_{b}\right)$ can be identified with the complement of some compact set in the Hyperbolic space $\left(\mathbb{H}^{n+1}, g_{\mathbb{H}}\right)$ realized as the hypersurface

$$
\left\{\left.\left(x_{1}, \ldots, x_{n+1}, t\right)|| x\right|^{2}-t^{2}=-1, t>0\right\} \subset \mathbb{R}^{n+1,1}
$$

by letting $\sinh r=\rho=\frac{1}{|x|}$. The restriction of $t$ to $\mathbb{H}^{n+1}$ is an eigenfunction of $\left(\mathbb{H}^{n+1}, g_{\mathbb{H}}\right)$, i.e.

$$
\Delta_{\mathbb{H}} t=(n+1) t .
$$

Moreover, as observed in [12], by a change of variables,

$$
t=\frac{1+|y|^{2}}{1-|y|^{2}}
$$


and $\left(\mathbb{H}^{n+1}, g_{\mathbb{H}}\right)=\left(B^{n+1},\left(\frac{2}{1-|y|^{2}}\right)^{2}|d y|^{2}\right)$, which tells us $(t+1)^{-2} g_{\mathbb{H}}$ compactifies $\mathbb{H}^{n+1}$ to be the standard Euclidean ball $\bar{B}^{n+1} \subset \mathbb{R}^{n+1}$ with totally umbilical boundary $S^{n}$. This, leads us to transplant $t$ to the domain $U$ and then look for a positive eigenfunction $u$ on $\left(X^{n+1}, g\right)$ which behaves like $t$ near $M$. To simplify notations, we use $v \in O_{k}\left(\rho^{s}\right)$ to standard for $v \in \Lambda_{k, \beta}^{s}(X)$ for $k \geq 0$ and a fixed $0<\beta<1$.

Lemma 4.1. There exists a smooth function $u>0$ on $(X, g)$ such that

$$
-\triangle_{g} u+(n+1) u=0
$$

and

$$
u=t+O_{2}\left(\rho^{\tilde{s}}\right)
$$

for some $1<\tilde{s}<n+1$.

Proof. The fact $r \in C^{2}(U)$ and $t=\sqrt{1+\frac{1}{\rho^{2}}}$ implies $t \in C^{2}(U)$. We calculate

$$
\begin{aligned}
-\triangle_{g} t & =-\frac{\rho^{n+1}}{\sqrt{\operatorname{det} g_{r}}} \partial_{r}\left(\rho^{1-n} \sqrt{\operatorname{det} g_{r}} \partial_{r} t\right) \\
& =-(n+1) t+\frac{1}{2} \operatorname{Tr}_{g_{r}} g_{r}^{\prime},
\end{aligned}
$$

where " " denotes differentiation with respect to $r$. By the decay assumption (1.2), we may choose $1<\tilde{s}<\min (s, n+1)$ such that $\operatorname{Tr}_{g_{r}} g_{r}^{\prime}=O_{0}\left(\rho^{\tilde{s}}\right)$. Hence, by Lemma 3.2, we know there exists a function $w=O_{2}\left(\rho^{\tilde{s}}\right)$ such that

$$
-\triangle_{g} w+(n+1) w=-\triangle_{g} t+(n+1) t .
$$

Let $u=t-w$. Then, $u>0$ by the maximum principle and the smoothness of $u$ follows directly from the local elliptic regularity theory.

We refer readers to [6], [12] and [3] for more results on eigenfunctions for asymptotically hyperbolic manifolds. In our next lemma, we set the stage to apply the work from [9] by using the eigenfunctions to compacitify the weakly asymptotically hyperbolic manifolds.

Lemma 4.2. The metric $g_{u}=\frac{1}{(1+u)^{2}} g$ extends to a $C^{2}$ metric on $\bar{X}$ such that $g_{u}$ has non-negative scalar curvature in $X,\left(M,\left.g_{u}\right|_{T M}\right)$ is isometric to $\left(S^{n}, h_{0}\right)$ and the mean curvature of $M$ in $\left(\bar{X}, g_{u}\right)$ identically equals the constant $n$. 
Proof. First, we calculate the scalar curvature of $g_{u}$,

$$
\begin{aligned}
R\left(g_{u}\right) & =\frac{4 n}{1-n}(u+1)^{\frac{n+3}{2}}\left[\triangle_{g}-\frac{n-1}{4 n} R(g)\right](u+1)^{\frac{1-n}{2}} \\
& =-n(n+1)|d u|_{g}^{2}+2 n(n+1) u(u+1)+R(g)(u+1)^{2} .
\end{aligned}
$$

Since $\operatorname{Ric}(g) \geq-n g$, we have $R(g) \geq-n(n+1)$ so (4.6) implies

$$
\begin{aligned}
R\left(g_{u}\right) & \geq-n(n+1)|d u|_{g}^{2}+2 n(n+1) u(u+1)-n(n+1)(u+1)^{2} \\
& =n(n+1)\left(u^{2}-|d u|_{g}^{2}-1\right) .
\end{aligned}
$$

As in [12], we then appeal to the Bochner formula for eigenfunctions, which is observed in [6].

$$
\begin{aligned}
\triangle_{g}\left(|d u|_{g}^{2}-u^{2}\right) & =2 n|d u|_{g}^{2}+2 \operatorname{Ric}\left(\nabla_{g} u, \nabla_{g} u\right)+2 \mid \text { Hess }\left._{g} u\right|_{g} ^{2}-2(n+1) u^{2} \\
& \geq 2\left|H e s s_{g} u\right|_{g}^{2}-2(n+1) u^{2}
\end{aligned}
$$

where the last step holds again since $\operatorname{Ric}(g) \geq-n g$. Therefore, we have

$$
-\triangle_{g}\left(u^{2}-|d u|_{g}^{2}-1\right) \geq 2\left|H e s s_{g} u-u g\right|_{g}^{2} .
$$

Hence, in order to prove the scalar curvature $R\left(g_{u}\right) \geq 0$, we only need to apply a maximum principle to $u^{2}-|d u|^{2}-1$ and verify that it goes to zero towards the boundary. A straightforward calculation reveals that

$$
\begin{aligned}
u^{2}-|d u|_{g}^{2}= & t^{2}-2 t w+w^{2}-\rho^{2}\left(\partial_{r} t\right)^{2}-\rho^{2} 2 \partial_{r} t \partial_{r} w \\
& -\rho^{2}\left(\partial_{r} w\right)^{2}-g^{\delta \lambda} \partial_{\delta} w \partial_{\lambda} w \\
= & +O_{2}\left(\rho^{\tilde{s}-1}\right)+O_{2}\left(\rho^{2 \tilde{s}}\right)+O_{1}\left(\rho^{\tilde{s}-1}\right) \\
& +O_{1}\left(\rho^{2 \tilde{s}}\right)+O_{1}\left(\rho^{2 \tilde{s}}\right)
\end{aligned}
$$

where, we have used the fact $t^{2}-\rho^{2}\left(\partial_{r} t\right)^{2}=1$. It follows from $\tilde{s}>1$ that

$$
u^{2}-|d u|_{g}^{2}-1 \rightarrow 0, \text { as } \rho \rightarrow 0
$$

Thus, we have

$$
u^{2}-|d u|_{g}^{2}-1 \geq 0 \text { on } X,
$$

which implies $R\left(g_{u}\right) \geq 0$ on $X$ by (4.7). 
Next, we consider the expansion of $g_{u}$ near $M$,

$$
g_{u}=\frac{1}{[(u+1) \rho]^{2}}\left\{d r^{2}+g_{r}\right\}
$$

where

$$
(u+1) \rho=\cosh r+\sinh r-w \sinh r .
$$

Since $w=O_{2}\left(\rho^{\tilde{s}}\right), \tilde{s}>1$ and $\rho^{2} g=d r^{2}+g_{r}$ is $C^{2}$ on $\bar{X}$, we see that $g_{u}$ readily extends to a $C^{2}$ metric on $\bar{X}$. Furthermore, we have the boundary values

$$
\left.(u+1) \rho\right|_{r=0}=1 \text { and }\left.\frac{d}{d r}[(u+1) \rho]\right|_{r=0}=1,
$$

which, combined with the facts $g_{0}=h_{0}$ and $T r_{g_{0}} g_{0}^{\prime}=0$, show that $\left(M, g_{u} \mid T M\right)$ is isometric to $\left(S^{n}, h_{0}\right)$ and $M$ has constant mean curvature $n$ in $\left(\bar{X}, g_{u}\right)$.

Now it follows from Proposition 3.3 that $R\left(g_{u}\right) \equiv 0$ on $X$. (4.7), (4.12) and (4.9) then imply that

$$
\left|H e s s_{g} u-u g\right|=0 .
$$

Therefore, $\left(X^{n+1}, g\right)$ is the hyperbolic space $\mathbb{H}^{n+1}$ by Lemma 3.5 .

Remark 4.3. We may reformulate the decay assumption (1.2) in terms of the metric expansion (3.1). By substituting, $s=\frac{\cosh (r)-1}{\sinh (r)}$ back, we see that (1.2) is equivalent to

$$
\left(1-\frac{s^{2}}{4}\right) \operatorname{Tr}_{g_{s}}\left(\frac{d}{d s} g_{s}\right)+n s \in \Lambda_{0, \beta}^{\delta}(X)
$$

for some $\delta>1$.

To conclude, we would like to make some remarks. The main theorem in this paper improves the rigidity theorem in [12]. Here, we no longer assume that the conformally compact manifolds are Einstein and we assume much weaker asymptotics at the infinity. In other words, we only assume the Einstein equations are satisfied at the infinity to a very low order, which is often true since the energy-momentum tensor usually vanishes to certain order for isolated systems. Also, we believe it is interesting to compare our result to the scalar curvature rigidity for asymptotically hyperbolic manifolds in [1], [4], [10], [16]. For example, in [15], Wang defines a conformally compact manifold $\left(X^{n+1}, g\right)$ to be asymptotically hyperbolic if it satisfies: 
1. $\left(X^{n+1}, g\right)$ is weakly asymptotically hyperbolic with the conformal infinity being the standard sphere $\left(S^{n}, h_{0}\right)$.

2. Let $r$ be the special defining function so that we can write

$$
g=\frac{1}{\sinh ^{2}(r)}\left\{d r^{2}+g_{r}\right\}
$$

in a neighbourhood of $\partial X$. Then

$$
g_{r}=h_{0}+\frac{r^{n+1}}{n+1} h+O\left(r^{n+2}\right),
$$

where $h$ is a symmetric 2-tensor on $S^{n}$. Moreover, the asymptotic expansion can be differentiated twice.

Working with this definition, see also [1], [4] and [10], Wang was able to prove that if $\left(X^{n+1}, g\right)$ is asymptotically hyperbolic, $\left(X^{n+1}, g\right)$ is spin and the scalar curvature $R \geq-n(n+1)$, then

$$
\int_{S^{n}}\left(\operatorname{Tr}_{h_{0}} h\right) d \mu_{h_{0}} \geq\left|\int_{S^{n}}\left(\operatorname{Tr}_{h_{0}} h\right) x d \mu_{h_{0}}\right|
$$

Moreover, equality holds if and only if $(X, g)$ is isometric to the hyperbolic space $\mathbb{H}^{n+1}$. Since, our decay assumption in Theorem 1.1 is much weaker than (4.19), we immediately have the following corollary,

Corollary 4.4. Let $\left(X^{n+1}, g\right)$ be an asymptotically hyperbolic manifold in the sense of [15]. If $2 \leq n \leq 6$ and Ric $\geq-n g$, then $\left(X^{n+1}, g\right)$ is isometric to the hyperbolic space $\mathbb{H}^{n+1}$.

\section{References.}

[1] Lars Andersson and Mattias Dahl. Scalar curvature rigidity for asymptotically locally hyperbolic manifolds. Ann. Global Anal. Geom. 16(1) 1998, 1-27.

[2] Robert Bartnik. The mass of an asymptotically flat manifold. Comm. Pure Appl. Math. 39(5) 1986, 661-693.

[3] Sun-Yung A. Chang, Jie Qing, and Paul Yang. On the topology of conformally compact einstein 4-manifolds. preprint, http://arXiv.org/abs/math.DG/0305085, 2003. 
[4] P. Chrusciel and M. Herzlich. The mass of asymptotically hyperbolic riemannian manifolds. preprint math. DG/0110035.

[5] C. Robin Graham and John M. Lee. Einstein metrics with prescribed conformal infinity on the ball. Adv. Math. 87(2) 1991, 186-225.

[6] John M. Lee. The spectrum of an asymptotically hyperbolic Einstein manifold. Comm. Anal. Geom. 3(1-2) 1995, 253-271.

[7] Mario Listing. Sectional curvature rigidity of asymptotically hyperbolic manifolds. preprint. 2003.

[8] Rafe Mazzeo. The Hodge cohomology of a conformally compact metric. J. Differential Geom. 28(2) 1988, 309-339.

[9] Pengzi Miao. Positive mass theorem on manifolds admitting corners along a hypersurface. Adv. Theor. Math. Phys. 6(6) 2002, 1163-1182.

[10] Maung Min-Oo. Scalar curvature rigidity of asymptotically hyperbolic spin manifolds. Math. Ann. 285(4) 1989, 527-539.

[11] Jie Qing. On the uniqueness of ads space-time in higher dimensions. preprint 2003.

[12] Jie Qing. On the rigidity for conformally compact Einstein manifolds. Int. Math. Res. Not. (21) 2003, 1141-1153.

[13] Richard Schoen and Shing Tung Yau. On the proof of the positive mass conjecture in general relativity. Comm. Math. Phys. 65(1) 1979, 45-76.

[14] Yuguang Shi and Luen-Fai Tam. Positive mass theorem and the boundary behaviors of compact manifolds with non-negative scalar curvature. J. Differential Geom. 62(1) 2002, 79-125.

[15] Xiaodong Wang. The mass of asymptotically hyperbolic manifolds. J. Differential Geom. 57(2) 2001, 273-299.

[16] Xiao Zhang. Positive mass theorem in general relativity. preprint 2001. 